\title{
GROWTH, ALLOMETRY, SHAPE-VARIATION AND INSTAR DISCRETION IN AGNOSTOID TRILOBITES
}

HUNT*, Gene, Research Training Program, National Museum of Natural History, Smithsonian Institution, Washington, DC 20560, U.S.A.; CHAPMAN, Ralph E., Applied Morphometrics Laboratory, ADP NHB MRC 136, National Museum of Natural History, Smithsonian Institution, Washington, DC 20560, U.S.A.; HUGHES, Nigel C., Geier Collections and Research Center, Cincinnati Museum of Natural History, 1720 Gilbert Avenue, Cincinnati, OH 45202, U.S.A.

Agnostoid trilobites exhibit relatively simple cephalic and pygidial morphologies that lend themselves to morphometric and allometric analyses. Despite this, relatively little quantitative work has been done on agnostoid growth and variation. The one notable exception is the study by A.S. Hunt on the Ordovician species Trinodus elspethi where he studied its growth, allometry and instars in detail using bivariate methods. This study showed $T$. elspethi as exhibiting almost no change in shape during growth and very discrete instars, more typical of ostracodes, for both the cephalon and pygidium. If these conservative patterns of shape change are typical for agnostoids in general then they would be excellent subjects for evolutionary analyses and would make them especially interesting for morphospace studies. If discrete instars are common, they also would be very useful for studies of size variation within ecological and biogeographical contexts and in studying the effects of taphonomic time-averaging.

As part of a long-term project analyzing growth, allometry and shape variation in agnostoids we have analyzed a number of growth series of different species, including the Cambrian forms Agnostus pisiformis and Peronopsis interstricta, and re-analyzed the data collected by Hunt for T. elspethi from different localities. We have used the bivariate methods applied by Hunt, as well as multivariate techniques and shapeanalysis methods developed over the last 10 years, including RFTRA, Booksteinian Shape Coordinates and Thin Plate Splines. Morphometric data were collected as coordinates using an image analysis system and then analyzed.

The results show that all agnostoids analyzed so far exhibit very conservative trends in their shape variation, although subtle but consistent trends are apparent. This is apparent, for the most part, even if different localities are sampled for the same taxon, although additional variation is introduced by this. This suggests agnostoids are a perfect group to study using morphospace approaches because the individual taxa will act as very discrete units occupying small regions of a morpho- space, even if full growth series are included. The data also suggest that discrete instars may also be uncommon among agnostoids, perhaps due to taphonomic time-averaging, and that the results reported for T. elspethi are not typical for that group. The data for T. elspethi, from all available localities, are being re-analyzed and some specimens re-measured to determine just how common discrete instars are in that species. 